\title{
REVIEW \\ Central motor conduction studies in patients with spinal cord disorders: a review
}

\author{
R Nardone ${ }^{1,2,3}$, Y Höller ${ }^{1,3}$, A Thomschewski ${ }^{1,3}$, P Höller $^{1,3}$, J Bergmann ${ }^{4}$, S Golaszewski ${ }^{1}$, \\ F Brigo ${ }^{2,5}$ and E Trinka ${ }^{1,3}$
}

Study design: Topical review of the literature.

Objectives: The evaluation of patients with myelopathies requires radiological investigations; however, for the correct interpretation of the neuroimaging findings, the functional assessment of corticospinal conduction is helpful or even mandatory in many conditions. The objective of this review article was to assess the utility of the motor evoked potentials (MEPs) in diagnosis and management of the most frequent spinal cord disorders.

Setting: Salzburg (Austria) and Merano (Italy).

Methods: A MEDLINE search was performed using following terms: 'motor evoked potentials', 'transcranial magnetic stimulation', 'central motor conduction', 'compressive myelopathy', 'spinal cord infarction', 'spinal cord injury', 'syringomyelia', 'myelitis', 'hereditary spastic paraparesis', 'subacute combined degeneration' and 'hepatic myelopathy'.

Results: Central motor conduction abnormalities can be detected also in the absence of neuroradiological abnormalities-for example, in patients with subacute combined degeneration or hepatic myelopathy. In the most frequent patients with compressive myelopathies, MEPs were found to be very helpful in determining the functional significance of neuroimaging findings. MEP recording can supplement clinical examination and neuroimaging findings also in the assessment of the spinal cord injury level. In patients with spinal cord infarction, the MEP study can demonstrate spinal involvement even when radiological evidence for spinal cord damage is absent or equivocal, thus allowing an important early diagnosis.

Conclusion: MEPs represent a highly sensitive and accurate diagnostic tool in many different spinal cord disorders. MEPs can also be useful in follow-up evaluation of motor function during treatment and rehabilitation.

Spinal Cord (2014) 52, 420-427; doi:10.1038/sc.2014.48; published online 22 April 2014

\section{INTRODUCTION}

Neuroradiological examination represents the procedure of choice for the diagnosis of myelopathies; however, a correct interpretation of the data provided by neuroimaging can be achieved only if morphological findings are correlated with functional data. ${ }^{1}$ The neurophysiological evaluation is essential to provide functional correlate to radiological abnormalities.

In particular, the motor evoked potentials (MEPs) elicited by transcranial magnetic stimulation (TMS), together with the somatosensory evoked potentials (SEPs), represent a highly accurate diagnostic test, and a very high sensitivity value has been demonstrated especially in spinal cord disorders. ${ }^{2}$

The determination of central motor conduction time (CMCT) is a sensitive method to detect myelopathies, and abnormalities may be observed in the absence of radiological changes. ${ }^{3}$

MEP studies can help to localize the site of main interest for the neuroradiological study or in detecting a subclinical involvement of central motor pathways.

For neurologists and neurosurgeons having to identify the causes of acute spinal symptoms - for example, disc herniation with cord compression, cervical canal stenosis, multiple sclerosis, transverse myelitis, spinal contusion or bleeding, vascular malformations or spinal tumors - the differential diagnosis is often difficult and MEPs may provide an important adjuvant diagnostic tool.

The present review is focussed on studies that assessed, among the measures of corticospinal projection, the value of CMCT measurement in patients with the most important spinal cord disorders.

\section{MATERIALS AND METHODS}

\section{Search strategy}

The MEDLINE search, accessed by Pubmed (1966-November 2013) and EMBASE (1980-November 2013) electronic databases, was performed using the medical subject headings 'motor evoked potentials', 'transcranial magnetic stimulation', 'central motor conduction', as well as following free terms, combined in multiple search strategies with Boolean operators in order to find relevant articles: 'compressive myelopathy', 'spinal cord infarction', 'spinal cord injury', 'syringomyelia', 'hereditary spastic paraparesis', 'subacute combined sclerosis', 'hepatic myelopathy' and 'myelitis'.

Two review authors ( $\mathrm{YH}$ and $\mathrm{AT}$ ) screened the titles and abstracts of the initially identified studies to determine whether they satisfied the selection criteria, independently assessed the methodological quality of each study and

${ }^{1}$ Department of Neurology, Christian Doppler Klinik, Paracelsus Medical University, Salzburg, Austria; ${ }^{2}$ Department of Neurology, Franz Tappeiner Hospital, Merano, Italy; ${ }^{3}$ Spinal Cord Injury and Tissue Regeneration Center, Paracelsus Medical University, Salzburg, Austria; ${ }^{4}$ Department of Psychology and Center for Neurocognitive Research, University of Salzburg, Salzburg, Austria and ${ }^{5}$ Section of Clinical Neurology, Department of Neurological, Neuropsychological, Morphological and Movement Sciences, University of Verona, Verona, Italy

Correspondence: Dr R Nardone, Department of Neurology, Franz Tappeiner Hospital, via Rossini, 5 Merano (BZ), Merano 39012, Italy.

E-mail: raffaele.nardone@asbmeran-o.it

Received 4 December 2013; revised 17 March 2014; accepted 22 March 2014; published online 22 April 2014 
risk of bias, focusing on blinding and other potential sources of bias. Case reports or patient small series were also included. Some studies were conducted by groups with common authors, so that the probability of overlapping cases and/or controls cannot be ruled out. It was therefore impossible to determine whether these included papers that represent duplicate publications of one study or two separate studies (multiple publication bias). The search strategy described above yielded 58 papers, the earliest was published in 1988 and the most recent in 2013.

\section{MEP and CMCT}

CMCT estimates the conduction time of corticospinal fibers between motor cortex and spinal (or bulbar) motoneurons. ${ }^{3}$ It includes the times for excitation of cortical cells, conduction via the corticobulbar-corticospinal tract and excitation of the motoneuron sufficient to reach its firing threshold. The practical aspects of CMCT measurement in a clinical setting are covered in a recent review article. ${ }^{4}$ CMCT is calculated by subtracting the peripheral conduction time from the MEP latency recorded after cortical stimulation. The peripheral conduction time may be estimated by two methods. First, peripheral conduction time is calculated as $(\mathrm{F}+\mathrm{M}-1) / 2$, where $F$ is the shortest F-latency and $M$ is the $\mathrm{M}$-wave latency. ${ }^{5}$ However, this method is only applicable in relatively distal muscles. The shortest F-latency is easier to measure and has been used to develop normal ranges in most studies. The second approach to calculate the peripheral conduction time is to stimulate either electrically or magnetically over the vertebral column; this procedure excites motor roots at their exit foramina. ${ }^{5}$ However, this method overestimates the real CMCT because the conduction time in proximal root segment between cord and exit foramen is included.

CMCT is usually measured with the active target muscle. In this situation there is the greatest opportunity for the descending corticospinal volley to cause a discharge because the spinal motor neuron pool is close to firing threshold. For CMCT measurement, the MEP latency is the only consideration, and with a contraction of 10-20\% maximum background force, latency variation is much less than amplitude variation. ${ }^{5}$ It is acceptable to record five responses and then measure the shortest latency. ${ }^{6}$

The motor root conduction time is the conduction time from motoneuron cell body to root exiting at the neural foramen. Motor root conduction time can be calculated as the latency difference between the peripheral conduction time estimated by the stimulation method and that estimated by the F-wave method. ${ }^{7}$

Lengthening of CMCT suggests demyelination along the motor pathways, whereas low-amplitude MEPs with little delay or absence of responses suggest neuronal or axonal loss. ${ }^{8}$ The amplitude of the MEP reflects integrity of the corticospinal tract and excitability of motor cortex and spinal levels, as well as conduction along the peripheral motor pathway to the muscles. ${ }^{8}$

\section{RESULTS}

\section{Compressive myelopathy}

Cervical spondylotic myelopathy. A number of studies have demonstrated that almost all patients with cervical spondylotic myelopathy (CSM) present an MEP abnormality for distal upper limb muscles and for lower limb muscles. ${ }^{9-19}$ For careful assessment of the cervical spinal cord, MEPs should be recorded from multiple upper limb muscles corresponding to different segmental levels. ${ }^{18}$

In patients with a high cervical cord compression, the CMCT is prolonged for biceps brachii and distal hand muscles. Conversely, in patients with multilevel cervical cord compression such as cervical canal stenosis, CMCT was found to be abnormal for thenar muscle and always normal for the biceps muscle. ${ }^{18}$ This finding suggests a prevalent damage of the central motor pathways to distal upper limb muscles. In other patients, the CMCT abnormality is confined to distal upper limb muscles, and these have usually a single compression level such as a central disc herniation.

In patients showing a close correlation between neuroradiological and neurophysiological findings, a segmental demyelination of central motor pathways because of a direct mechanical cord compression is probably the most important etiologic factor. However, there are sometimes discrepancies between the level of spinal cord compression documented by magnetic resonance imaging (MRI) and the level of spinal cord dysfunction revealed by MEPs. In these cases, more than one mechanism of spinal cord damage may have a role in the pathogenesis of CSM.

In particular, a more caudal electrophysiological involvement of the cervical spinal cord can be explained on the basis of other mechanisms, such as a compromised blood flow to the cord. The frequent involvement of lower cervical segments may depend on their higher vulnerability to ischemic damage. In fact, the anterolateral regions of lower cervical segments are the regions where blood supply is almost exclusively from the anterior spinal artery, whereas the higher cervical segments are located between the cervical and intracranial arterial territories and have therefore more sources of blood supply. When cervical spondylotic compression involves the anterior spinal artery, the major damage is of vascular origin and is localized to the lower cervical segments, independent of the level of spondylotic degenerative changes. ${ }^{1}$

It should also be considered that other neurological disorders such as neoplastic compression, intraspinal tumors, myelitis, neuromyelitis optica (Devic's disease) and, even rarely, motoneuron disease (MND) may result in a similar electrophysiological pattern.

About $15 \%$ of CSM cases may present with an apparently isolated spastic paraparesis. In these patients, the MEP study may reveal a subclinical involvement of corticospinal projections to myotomes that are innervated by nerve roots of the cervical spinal cord. ${ }^{18}$ The abnormality of central motor conduction for distal upper limb muscles and for all muscles supplied by more caudal myelomeres in the presence of a normal CMCT for biceps muscles is frequently observed in CSM. When this pattern of MEP abnormality is recorded, MRI examination of the cervical spinal cord should be performed. On the contrary, an abnormal CMCT for the biceps muscle may rather indicate several other conditions because this finding can be observed in a high cervical cord compression and also in an intracranial disorder involving central motor pathways (such as stroke, normal pressure hydrocephalus, multiple sclerosis, diffuse axonal injury and cerebral palsy), MND or a neurodegenerative disorder involving the spinal cord.

The differential diagnosis between a high cervical cord lesion and an intracranial disorder is often clinically and electrophysiologically difficult. In this regard, it may be very helpful in the study of central conduction for more cranial myotomes, in particular MEPs of the trapezius muscle.

CMCT to the trapezius muscle is within normal limits in subjects with high cervical cord compression.

Besides the localization of the site of lesion, MEPs may provide valuable information in quantifying the degree of functional involvement of the spinal cord, in particular when MRI shows only a minor spondylogenic compression of the spinal cord, which can have no significant functional effect on the spinal cord. In a recent study, ${ }^{20}$ the level of spinal cord compression was determined according to the pattern of CMCT prolongation and compared with the level disclosed by MRI. The actual level of compression was correctly indicated by transcranial magnetic stimulation (TMS) in $87.5 \%$ of cases and by MRI in $12.5 \%$ of the cases. MEP recording may help in the identification of patients with severe dysfunction of central motor pathways that are probably better candidates for surgery. Moreover, serial MEP recording may be useful in identifying the progressive forms of CSM and in selecting patients who may benefit from surgical 
treatment. MEPs may be useful in determining the surgical site in patients who have multilevel disc herniations. Successively, MEPs can also have a role in monitoring the effect of surgical therapy. The average sensitivity of MEPs in CSM was 0.82 .

In a retrospective study, Capone et al. ${ }^{21}$ used MEPs for the functional assessment of the spinal cord before and after surgery and correlated MEP changes with clinical findings. The authors found that early surgical intervention for CSM could produce a beneficial effect on spinal cord functionality that can be detected by MEPs. Clark et al. ${ }^{22}$ also reported a correlation between decreased intraoperative MEPs and postoperative new neurological deficits in patients with CSM.

Thoracic/lumbar spinal cord compression. In patients with spinal cord compression at the thoracic level, MEP study shows a normal CMCT for upper limb muscles and abnormal conduction for the lower limb muscles.

To assess thoracic cord conduction, responses can also be recorded from intercostal muscles. ${ }^{23,24}$ The study of different muscles innervated by the thoracic cord, such as the rectus abdominis or external oblique muscles, may be useful because the roots of these muscles can be stimulated using magnetic stimulation and CMCT to the corresponding thoracic segments can be measured.

In addition, the clinical diagnosis of lumbosacral cord lesions may be difficult. In fact, upper motoneuron signs may be masked by the prevalence of lower motoneuron involvement, thus leading to a misdiagnosis of cauda equina syndrome. Similarly, in patients affected by MND, lower MND can confound the interpretation of the testing of upper MND. Conversely, lower motoneuron signs may be absent or very mild because of the prevalence of central motor pathway involvement.

MEP recording from multiple lower limb muscles, corresponding to different cord levels, is useful in localizing the site of the lesion. The double determination of CMCT can help in differentiating a slowing along central motor pathways from a delay along lumbosacral motor roots. ${ }^{25}$ The RMCT can be evaluated from the difference between the two differently calculated central conduction times. When CMCT (calculated with both methods) and RMCT are prolonged, a myeloradicular lumbosacral lesion is highly probable. On contrary, an abnormal CMCT for distal lower limb muscles confirmed by the F-wave method in association with normal RMCT and normal CMCT for more proximal lower limb muscles indicates that the lesion is localized at the lowest part of the lumbosacral cord. An abnormal CMCT evaluated with both methods, associated with a prolonged RMCT, strongly suggests a dysfunction of multiple lumbosacral roots and a normal spinal cord and can be observed also in patients with neurogenic claudication due to lumbar canal stenosis.

\section{Spinal cord vascular disorders}

The evaluation of patients with suspected spinal cord infarction requires clinical assessment and radiological investigations. The clinical diagnosis is often difficult, also because in the early phases of spinal cord ischemia MRI can be unrevealing. ${ }^{26}$

Imaging (MRI) of spinal cord ischemia is well established; however, its use in the acute setting can be difficult. In spinal cord infarction diffusion-weighted MRI of the spinal cord is rarely performed because of several technical issues, including the small size of the spinal cord. Usually, only follow-up conventional MRI examinations (at least several hours after clinical onset) show medullary pathological findings.

Nardone et al. ${ }^{27}$ explored in one case each of cervical and lumbar spinal cord infarction whether MEP examination may contribute to early diagnosis. Spinal MRI at admission was unremarkable in both patients. At this time, MEPs were abnormal in both patients, to the upper and lower limbs in the first patient, exclusively to the lower limbs in the second (Figure 1). Follow-up MRI examinations documented an infarction in the territory of the anterior spinal artery and of the Adamkiewicz artery, respectively.

MEP study can thus be useful in demonstrating spinal cord involvement also when radiological evidence for spinal cord damage is absent or equivocal. Early diagnosis may allow earlier intervention and contribute to improved patient management.

The contribution of MEPs was found to be relevant also for spinal disorders resulting from dural arterovenous malformation, for which the clinical diagnosis may be very difficult. ${ }^{28}$ Indeed, together with a spinal cord lesion, the venous congestion frequently causes radicular involvement, and in these cases the clinical manifestation is characterized by a preponderance of lower motoneuron signs and pain, thus mimicking a lumbar canal stenosis. Moreover, as spondylotic changes of the lumbosacral column are common in elderly subjects, the MRI study may lead to an incorrect attribution of the cause of the disorder to spondylotic changes of the lumbosacral column. In these cases, MEPs are useful to document a lower spinal cord involvement, and the neuroradiological examination should be extended to this region.

Among other ischemic myelopathies, abnormal MEP findings have been also reported in the context of cocaine abuse. ${ }^{29,30}$ CMCT abnormalities have been observed following thoracoabdominal aortic surgery. ${ }^{31-33}$ Monitoring MEPs is considered a highly reliable
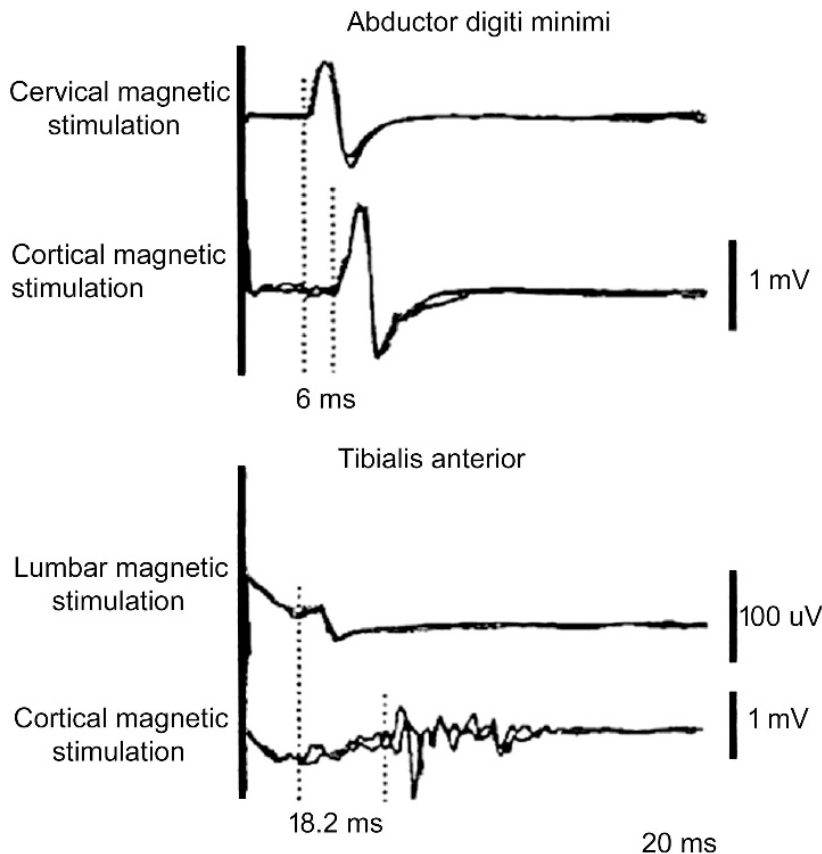

Figure 1 MEP of a patient with spinal cord infarction at the level T10-L1. Left abductor digiti minimi and left tibialis anterior records are shown. In the upper traces, the motor evoked responses after radicular stimulation are shown in the lower traces the evoked responses after magnetic stimulation of the motor cortex. CMCT values are indicated. Normal upper limits (mean +3 s.d.) are 7.7 and $17.1 \mathrm{~ms}$, respectively. The latencies of the responses obtained after cervical and lumbar stimulation are normal. CMCT to the upper limbs is normal, whereas CMCT to the lower limbs is prolonged. Reproduced from Nardone et al. ${ }^{27}$ 
technique to assess spinal cord ischemia during descending thoracic or thoracoabdominal aortic surgery.

\section{Syringomyelia}

Noques $^{34}$ first examined MEPs and SEPs in 13 patients with syringomyelia. Prolonged CMCT or absent motor responses in upper or lower limbs were found in most patients. MEP sensitivity was 0.77. Measurement of CMCT, followed by SEPs after tibial nerve stimulation, disclosed the greatest number of abnormalities. Two of five patients who went through surgery improved clinically and showed reduction in CMCT after surgical treatment. MEPs are thus useful in the evaluation of anterolateral spinal cord function. Masur et al. $^{35}$ investigated the relation of clinical symptoms, MRI findings and electrophysiological data (SEPs and MEPs) in 22 patients with syringomyelia. They reported a MEP sensitivity of 0.68 and found a close relation between clinical symptoms and electrophysiological data, while degree of disability and duration of the disease were not related to the dimensions of the syrinx. Both MEPs and SEPs detected subclinical deficits. In another study, MEP abnormalities have been reported in $44 \%$ of patients with syringomyelia. ${ }^{36}$ However, MEPs may be normal even in a patient with an extensive syrinx; therefore, a normal CMCT finding does not exclude this diagnosis, and the MEP study is of rather limited value for diagnosing and monitoring the course of syringomyelia.

\section{Heredodegenerative spinal cord disorders}

Hereditary spastic paraparesis. In the first studies either absent or clearly reduced or prolonged MEPs from the lower limbs were recorded in all patients with hereditary spastic paraparesis (HSP). ${ }^{37,38}$ Conversely, CMCT to upper limbs was found to be completely normal in most cases. Moreover, CMCT for lower limb muscles is usually only slightly prolonged, despite the often severe spasticity. This distribution of abnormalities, which suggests a differential involvement of the spinal pathways, may be explained on the basis of the major neuropathological feature of HSP represented by axonal degeneration that is maximal in the longer pyramidal fibers supplying the lower extremities. In fact, under all conditions characterized by an axonal type of involvement of central motor pathways (such as HSP) the loss of large diameter axons causes only a minor degree of conduction slowing along central motor pathways. CMCT abnormality becomes evident only for the lower limb long tracts, whereas CMCT is normal for upper limbs. In cases of axonal central motor disorders, the CMCT for lower limbs exceeds the normal upper limits by a few milliseconds, whereas in spinal cord compression the prolongation of CMCT to the lower limbs, which is caused by segmental demyelination of the spinal cord, is usually more pronounced. ${ }^{1}$ Even much delayed CMCT to lower limbs or absent cortical MEPs are usually observed in subjects with inflammatory demyelinating diseases of the central nervous system, such as multiple sclerosis. However, in a more recent study, MEPs were abnormal along the central pathway for both upper and/or lower limbs in all the four patients with HSP and a molecular diagnosis of SPG5. ${ }^{39}$ The severely prolonged CMCT to lower limbs supports a contemporary demyelinating damage of the corticospinal tracts in this phenotype. ${ }^{40}$ In another study, MEPs were recorded from upper and lower limb muscles in 12 patients affected by autosomal dominant HSP with spastin mutation (SPG4). ${ }^{41}$ In all cases, direct and indirect CMCT calculated by subtracting from the cortical latency those that were obtained on magnetic spinal stimulation and via the F-wave method were significantly longer than the controls.
The marked discrepancy between clinical and electrophysiological findings is peculiar to HSP and may be useful in the differential diagnosis with CSM. In fact, unlike HSP patients, in CSM subjects the CMCT to hand muscles may be abnormal even in patients with mild or absent pyramidal signs in the upper limbs.

\section{Myelitis}

In inflammatory disorders of the spinal cord such as acute transverse myelitis, MEPs can be used to localize the site of the lesion and, in some cases, may also show abnormalities even when MRI is unremarkable. In the study of Kalita and Misra ${ }^{42}$ the reported sensitivity was 1 . In a segmental myelitis at the thoracic level, the MEPs are normal for upper limbs and prolonged for lower limb muscles. The recording from paravertebral muscles shows a normal conduction to paraspinal muscles proximal to the lesion level, whereas no responses are recorded from more caudal paravertebral sites. The evaluation of motor conduction along the spinal cord and roots may help to define the exact distribution of the inflammatory process, and to establish whether the patients are affected by isolated myelitis, myeloradiculitis or isolated radiculitis. In cases of myeloradiculitis, CMCT to distal hand muscles is prolonged with both techniques of determination, and the root conduction time is also prolonged.

The demonstration of spinal cord involvement may also be useful in the diagnosis because in the early phases of the disease, an acute myelitis may rarely mimic the clinical features of an acute polyradiculitis. In some types of myelitis, such as myelopathy associated with acquired immunodeficiency syndrome (AIDS) and HAM/TSP (HTLV-I-associated myelopathy/tropical spastic paraparesis), symptoms and signs of spinal cord involvement may be masked by a concomitant neuropathy. In these cases, MEP abnormalities demonstrate cord involvement that is usually localized at the thoracic level. Moreover, MEP studies performed on patients with HAM/TSP support the involvement of the pyramidal tract mainly at lower levels, following a centripetal pattern, whereas in AIDS patients such an involvement is more prominent at brain levels following a centrifugal pattern. The authors concluded that, in the retrovirusassociated neurodegenerative disorders, these differences in corticospinal tract involvement of patients with AIDS and HAM/TSP may help to orient early neurorehabilitation measures. ${ }^{43}$

\section{Nutritional/metabolic myelopathies}

Subacute combined degeneration (vitamin B12 deficiency). The neurological manifestations of vitamin B12 deficiency primarily result from the degeneration of the posterior and lateral columns of the spinal cord. The functional exploration of the central motor pathways in subacute combined degeneration may reveal a spinal cord dysfunction even in the early stages of the disease when the clinical symptoms may be limited to brisk tendon reflexes. CMCT to upper and lower limb muscles was abnormal in the four patients examined by Di Lazzaro et al. ${ }^{44}$ In another study, damage to the central motor pathway was identified in four out of nine patients. ${ }^{45}$ Interestingly, while MEPs and median SEPs were normalized after replacement (as well as MRI abnormalities), tibial SEPs remained abnormal in most patients.

Hepatic myelopathy. Utku et al. ${ }^{46}$ performed a MEP study in two patients with spastic paraparesis and found the absence of cortical MEPs in upper and lower extremities. These patients with chronic liver diseases were diagnosed as having hepatic myelopathy (HM) also because all the other possible diagnoses were ruled out. HM may 
rarely occur in patients with cirrhosis and other chronic liver diseases; this complication is usually associated with overt liver failure and surgical or spontaneous porto-systemic shunt.

To determine the frequency and gravity of HM, Nardone et al. ${ }^{47}$ performed a study examining MEPs in 13 patients with liver cirrhosis associated with porto-systemic shunt.

Six patients with clinical signs of spinal cord involvement also exhibited markedly prolonged CMCT, whereas milder MEP abnormalities were found in four of the seven patients with normal clinical examination. The clinical and neurophysiological features of patients with slight MEP abnormalities improved after liver transplantation, whereas no changes were detected in patients with a more advanced stage of disease (and more severe MEP abnormalities).

The MEP findings therefore support the potential value of the evaluation of the CMCT in preclinical and early stages of HM. Patients who undergo liver transplantation with preclinical or early HM documented by the MEP study seem to have a greater likelihood of recovery (Figure 2). ${ }^{47}$ It is thus possible that MEP/CMCTs have greater sensitivity in detecting preclinical or early HM and in assigning a prognosis for recovery after liver transplantation. Although a larger study comparing the sensitivity, specificity and

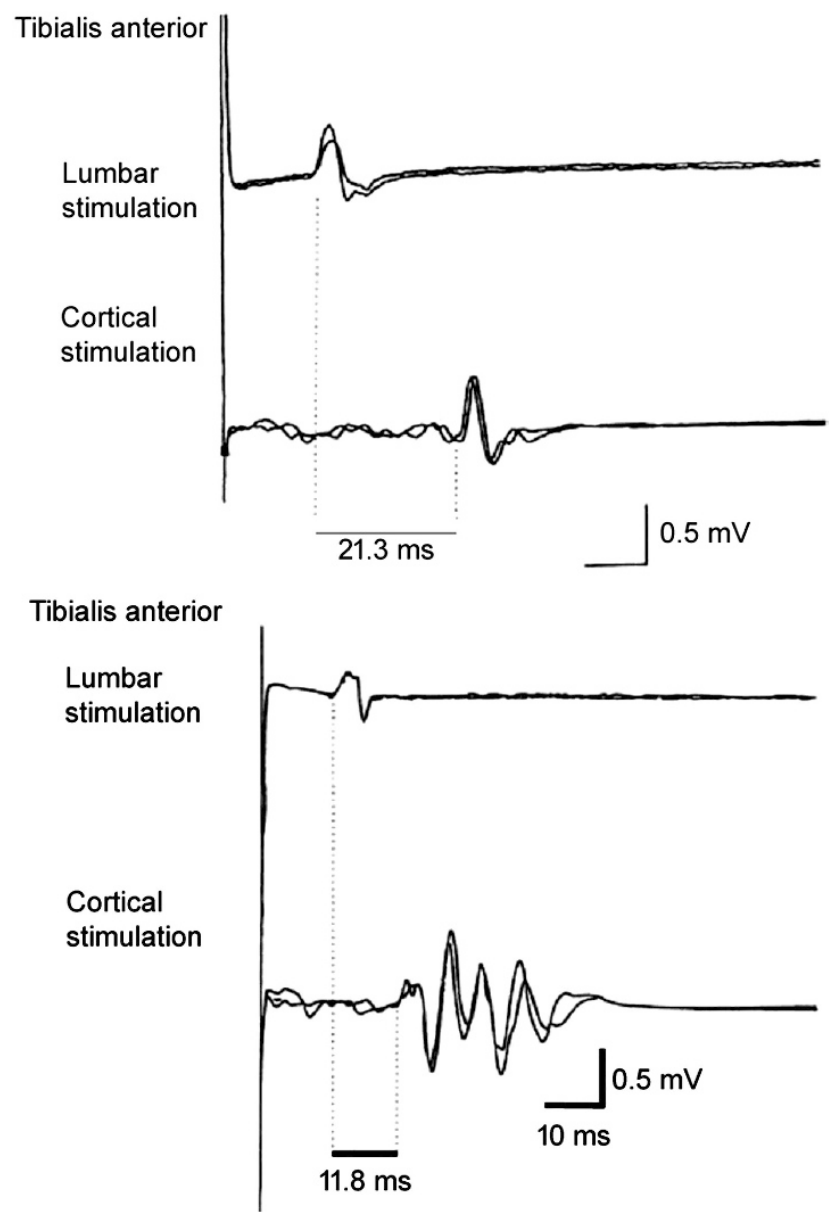

Figure 2 MEP evoked by lumbar and cortical stimulation in a patient with hepatic myelopathy before liver transplantation (LT; upper traces). CMCT values are indicated. A considerably abnormal CMCT prolongation can be observed. MEP evoked by lumbar and cortical stimulations after LT (lower traces). At this time normal CMCT can be recorded. Reproduced from Nardone et al. ${ }^{47}$ predictive value of MEP/CMCT is yet to be conducted, central motor conduction studies are an important consideration in the workup of patients with HM.

Nardone et ll $^{47}$ found an abnormal CMCT to the lower lumbar spinal segments and a normal CMCT to the upper cervical spinal segments, thus supporting localization to the thoracic spinal cord.
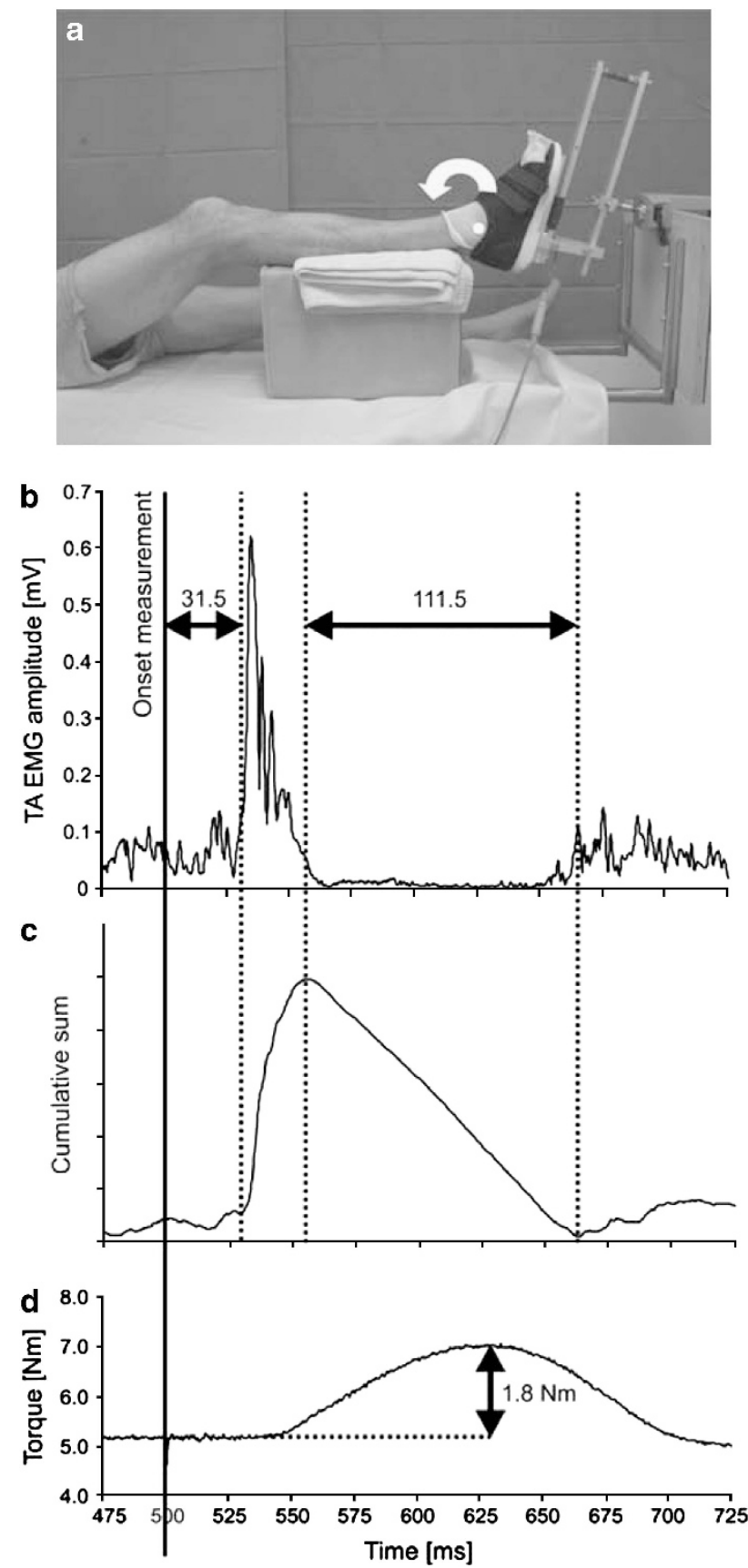

Figure 3 The figure illustrates a custom-made torque measurement device used to assess maximal voluntary isometric dorsal flexion torque (a), and the amount of background torque required for the transcranial magnetic stimulation (b-d). The analysis of MEP measures obtained from a healthy subject tested in the static condition at $20 \%$ maximal voluntary contraction The latency of the tibialis anterior EMG amplitude (b) was quantified using the cumulative sum (c). The difference between and the background torque and torque generated by the MEP response was also shown (d). Reproduced from van Hedel et al. ${ }^{55}$ 


\section{Spinal cord injury}

In patients with spinal cord injury (SCI), a good correlation was found between MEP findings and motor function. ${ }^{48,49}$ Interestingly, in SCI patients with high cervical lesion, MEPs can be recorded from the diaphragm, as well as from the scalenes, the parasternal intercostals and the expiratory rectus abdominis muscles, to investigate the central motor conduction properties of the respiratory muscles. ${ }^{50,51}$ Therefore, TMS can be used routinely to monitor patients with impaired central respiratory drive in SCI patients.

MEP recording can supplement clinical examination and neuroimaging findings in the assessment of the SCI level and can define the extent and the severity of pyramidal tract lesions in patients with SCI. The recording from multiple muscles can be used to identify the level of spinal cord lesion, and this is particularly helpful in uncooperative or unconscious patients. It should be considered that lower limb MEPs may be absent at rest even in a few normal subjects.

For this reason, the contribution of MEPs in the assessment of thoracolumbar SCIs is more limited than that for SCI at the cervical level. MEPs may be useful in the prediction of functional outcome in the acute phase of SCI. In a study of Curt et al., ${ }^{52}$ MEP recording from the abductor digiti minimi muscle was highly correlated to the outcome of hand function. ${ }^{52}$ When MEPs recorded from the abductor digiti minimi muscle were absent, active hand function was not regained. For the reasons previously explained, recordings of lower limb MEPs were less effective in predicting the recovery of ambulatory capacity, and there were some patients with an initial loss of lower limb MEPs who showed a recovery of ambulatory capacity. ${ }^{53}$

However, other authors found that assessment of MEPs of the anterior tibial muscle allows stratification of SCI according to lesion severity and outcome. The MEP amplitudes increased over 12 months after SCI, which was paralleled by a significant improvement of lower extremity motor scores and walking function. MEPs of the anterior tibial muscle latencies remained usually stable. ${ }^{54}$

Another study investigated the reliability of TA MEP measures controlled for dorsal flexion torque and motor task (Figure 3). ${ }^{55}$ The reliability of MEP amplitude was in general good. The reliability was good to excellent for MEP latency, maximal voluntary contraction and for the TMS threshold required to evoke a liminal MEP. The increased facilitation by the dynamic motor task showed the best reliability at $20 \%$ maximal voluntary contraction.

\section{DISCUSSION AND CONCLUSIONS}

In this narrative review, we have summarized studies demonstrating the usefulness of MEPs in the evaluation and management of many spinal cord disorders.

The first step in the diagnosis of spinal cord disorders and the main strategy for the correct management of the patients should be history taking and the clinical examination.

Nevertheless, MEP studies provide reliable information about the functional integrity and conduction properties of the corticospinal tracts in the diagnostic and prognostic assessment of various spinal cord disorders. It should be always considered that cortical stimulation produces both a direct corticospinal and indirect corticobulbar and then bulbospinal signal. As the latter is polysynaptic, the variably delayed monosynaptic signals that may emerge after injury or are present after treatment should be distinguished from those polysynaptic responses.

However, despite the limitations of the technique, MEPs allow following the evolution of motor disturbances control and to evaluate the effects of different therapeutic procedures.
The most frequently observed myelopathy is the CSM. The differential diagnosis between CSM and MND may be difficult because both conditions are characterized by a variable combination of upper and lower motoneuron signs. MEP studies-in particular, the multilevel assessment of the CMCT—can be extremely useful. In subjects with spinal cord compression, MEP abnormalities are recorded in all muscles localized below the level of the spinal lesion sparing muscles supplied by more cranial myelomeres. In MND, abnormal CMCT findings recorded from certain muscles may be associated with a normal CMCT to muscle innervated by more caudal myelomeres, resulting in a peculiar 'suspended' abnormality of the central motor conduction. Typically, in MND patients an abnormal CMCT for the biceps brachii may be associated with normal findings for distal upper and lower limb muscles. ${ }^{1}$

A possible explanation for this finding may be the restricted number of fast-conducting corticospinal projections to the biceps brachii, which were activated by TMS. Therefore, even the loss of a limited number of corticospinal fibers in the early stage of the disease may be sufficient to determine a CMCT abnormality for this muscle. It should be considered that a suspended abnormality of central motor conduction for biceps brachii can also be caused by a delay of conduction along the C5 and C6 roots. A concomitant lesion of these roots can be detected on clinical examination (that is, diminished biceps reflex) and/or electromyographic study of muscles innervated by $\mathrm{C} 5$ and $\mathrm{C} 6$ roots. On the contrary, a CMCT abnormality for proximal upper limb muscles with normal conduction to distal upper limb muscles is rarely observed in patients with CSM.1,19 The suspended central conduction abnormality can also be characterized by an abnormal CMCT for distal upper limb muscles associated with a normal conduction for lower limb muscles. In these cases, the evaluation of the central motor conduction with the F-wave method is mandatory for a correct localization of motor pathway dysfunction. MEP studies may also be particularly useful in the differential diagnosis between spinal cord compression and primary lateral sclerosis. This condition belongs to the group of MNDs, but only affects upper motoneurons, and can simulate a CSM when bulbar involvement is clinically not evident. However, the combination of MEP and clinical findings in the vast majority of patients with primary lateral sclerosis determines a very peculiar picture that can be of considerable value in the differential diagnosis. Indeed, MEPs are absent or extremely delayed, even in the early stage of the disease and in muscles with minimal weakness and/or spasticity. The marked alteration of MEPs evoked by cortical stimulation in association with a disproportionate minimal degree of motor deficit represents a pattern of MEP abnormalities that can be considered rather specific for primary lateral sclerosis.

In patients with spinal cord ischemia, MRI can be normal in the early phases and MEP study may be useful, along with clinical examination, in demonstrating spinal cord involvement and in localizing the site of central motor pathway dysfunction.

After hypoperfusion in the territory of the artery of Adamkiewicz, only lower limb MEPs are abnormal, whereas in case of an ischemic lesion in the territory of the anterior spinal artery, upper and lower limb MEPs are abnormal. MEPs may disclose spinal cord involvement even in the case of spinal transitory ischemic attacks.

On the other hand, diagnosis of lesions at the lumbar cord level is often difficult because clinical signs of upper motoneuron signs may be obscured by the prevalence of lower motoneuron impairment, thus causing a misdiagnosis of a cauda equine syndrome. Furthermore, lumbar spinal cord infarction may be associated with root damage due to venous congestion; in these cases, signs of lower motoneuron 
dysfunction and pain are the dominant clinical manifestations. It should be considered that the isolated finding of prolonged CMCT is not specific for any neurological disease, and that the facilitation of MEP responses usually induced by mild motor contraction is difficult or even impossible for the population of subjects with spinal cord disorders being partially or totally plegic. Although studies regarding motor functional recovery in these myelopathies have been promising, more trials are required to substantiate preliminary evidence.

In most patients affected by SCI or myelitis, spinal cord involvement is clinically evident, and the MEP contribution to the diagnosis is therefore mainly confirmatory. However, in these cases MEP studies may be useful in localizing levels of functional defects.

On the other hand, the functional outcome in traumatic SCI did not correlate with the strength of MEP signals. The motor output below the level of SCI is a function of both: the conduction of motor signals through the lesion (measured by MEPs) and the processing of those signals by neural circuitry below the level of injury (assessed, among others, by kimematic and poly-EMG studies). Many important functional aspects of motor control, such as coordination, speed of movement and sensory integration, may be affected by patterns of strength, spasticity (hypertonia, hyperreflexia and dysynergias) or sensory function/neuropathic pain (dysesthesias, hyperalgesia and allodynia).

Of great importance are MEP studies in patients with myelopathies associated with vitamin B 12 deficiency or chronic liver diseases. The findings of some studies indicate that the electrophysiological evaluation of central motor conduction may disclose an impairment of the corticospinal pathways even before the myelopathy is clinically manifested.

In conclusion, the above mentioned studies illustrate that the functional assessment of corticospinal conduction by means of MEP can significantly contribute in the diagnosis and management of spinal cord disorders and should not be overlooked by clinicians.

\section{DATA ARCHIVING}

There were no data to deposit.

\section{CONFLICT OF INTEREST}

The authors declare no conflict of interest.

1 Di Lazzaro V, Oliviero A. Evaluation of myelopathy, radikulopathy and thoracic nerve. In Hallett M and Chokroverty S (eds) Magnetic Stimulation in Clinical Neurophysiology. Elsevier, 2005, pp 105-127.

2 Di Lazzaro V, Oliviero A, Profice P, Ferrara L, Saturno E, Pilato F et al. The diagnostic value of motor evoked potentials. Clin Neurophysiol 1999; 110: 1297-1307.

3 Chen R, Cros D, Curra A, Di Lazzaro V, Lefaucheur JP, Magistris MR et al. The diagnostic utility of transcranial magnetic stimulation: report of an IFCN committee. Clin Neurophysiol 2008; 119: 504-532.

4 Groppa S, Oliviero A, Eisen A, Quartarone A, Cohen LG, Mall V et al. A practical guide to diagnostic transcranial magnetic stimulation: a report of an IFCN committee. Clin Neurophysiol 2012; 123: 858-882.

5 Mills KR. Magnetic Stimulation of the Human Nervous System. Oxford University Press: Oxford, 1999

6 Mills KR, Murray NM. Electrical stimulation over the human vertebral column: which neural elements are excited? Electroencephalogr Clin Neurophysiol 1986; 63: 582-589.

7 Banerjee TK, Mostofi MS, Us O, Weerasinghe V, Sedgwick EM. Magnetic stimulation in the determination of lumbosacral motor radiculopathy. Electroencephalogr Clin Neurophysiol 1993; 89: 221-226.

8 Hallett M. Transcranial magnetic stimulation and the human brain. Nature 2000; 406 $147-150$

9 Abbruzzese G, Dall'Agata D, Morena M, Simonetti S, Spadavecchia L, Severi P et al. Electrical stimulation of the motor tracts in cervical spondylosis. J Neurol Neurosurg Psychiatry 1988; 51: 796-802.

10 Maertens de Noordhout MA, Remacle JM, Pepin JL. Magnetic stimulation of the motor cortex in cervical spondylosis. Neurology 1991; 41: 75-80.
11 Herdmann J, Dvorak J, Bock WJ. Motor evoked potentials in patients with spina disorders: upper and lower motor neuron affection. Electromyogr Clin Neurophysio 1992; 32: 323-330.

12 Tavy DLJ, Wagner GL, Keunen RWM, Wattendorff AR, Hekster RE, Franssen H. Transcranial magnetic stimulation in patients with cervical spondylotic myelopathy: clinical and radiological correlations. Muscle Nerve 1994; 17: 235-241.

13 Chistyakov AV, Soustiel JF, Hafner H, Feinsod M. Motor and somatosensory conduction in cervical myelopathy and radiculopathy. Spine 1995; 20: 2135-2140.

14 Maertens de Noordhout AM, Myressiotis S, Delvaux V, Born JD, Delwaide PJ. Motor and somatosensory evoked potentials in cervical spondylotic myelopathy. Electroencephalogr Clin Neurophysiol 1998; 108: 24-31.

15 Bednarik J, Kadanka Z, Vohanka S, Novotný O, Surelová D, Filipovicová D et al. The value of somatosensory and motor evoked potentials in pre-clinical spondylotic cervical cord compression. Eur Spine J 1998; 7: 493-500.

16 Travlos A, Pant B, Eisen A. Transcranial magnetic stimulation for detection of preclinical cervical spondylotic myelopathy. Arch Phys Med Rehabil 1992; 73: 442-446.

17 Tavy DL, Franssen H, Keunen RW, Wattendorff AR, Hekster RE, Van Huffelen AC. Moto and somatosensory evoked potentials in asymptomatic spondylotic cord compression. Muscle Nerve 1999; 22: 628-634.

18 Di Lazzaro V, Restuccia D, Colosimo C, Tonali P. The contribution of magnetic stimulation of the motor cortex to the diagnosis of cervical spondylotic myelopathy. Correlation of central motor conduction to distal and proximal upper limb muscles with clinical and MRI findings. Electroencephalogr Clin Neurophysiol 1992; 85 311-320.

19 De Mattei M, Paschero B, Sciarretta A, Davini O, Cocito D. Usefulness of motor evoked potentials in compressive myelopathy. Electromyogr Clin Neurophysiol 1993; 33 205-216

20 Deftereos SN, Kechagias EA, Panagopoulos G, Seretis A, Orphanidis G, Antoniou E et al. Localization of cervical spinal cord compression by TMS and MRI. Funct Neurol 2009; 24: 99-105.

21 Capone F, Tamburelli FC, Pilato F, Profice P, Ranieri F, Di lorio R et al. The role of motor-evokes potentials in the management of cervical spondylotic myelopathy. Spine J 2013; 13: 1077-1079.

22 Clark AJ, Ziewacz JE, Safaee M, Lau D, Lyon R, Chou D et al. Intraoperative neuromonitoring with MEPs and prediction of postoperative neurological deficits in patients undergoing surgery for cervical and cervicothoracic myelopathy. Neurosurg Focus 2013; 35: E7.

23 Misawa T, Ebara S, Kamimura M, Tateiwa Y, Kinoshita T, Takaoka K. Evaluation of thoracic myelopathy by transcranial magnetic stimulation. J Spinal Disord 2001; 14: $439-444$

24 Chokroverty S, Deutsch A, Guha C, Gonzalez A, Kwan P, Burger R et al. Thoracic spinal nerve and root conduction: a magnetic stimulation study. Muscle Nerve 1995; 18 987-991

25 Di Lazzaro V, Pilato F, Oliviero A, Saturno E, Dileone M, Tonali PA. Role of motor evoked potentials in diagnosis of cauda equina and lumbosacral cord lesions. Neurology 2004 63: 2266-2271.

26 Weidauer S, Nichtweiss M, Lanfermann H, Zanella FE. Spinal cord infarction. MR imaging and clinical features in 16 cases. Neuroradiology 2002; 44: 851-857.

27 Nardone R, Bergmann J, Kronbichler M, Lochner P, Caleri F, Tezzon F et al. Magnetic resonance imaging and motor-evoked potential in spinal cord infarction. Neurol Sci 2010; 31: 505-509.

28 Linden D, Berlit P. Spinal arteriovenous malformations: clinical and neurophysiological findings. J Neurol 1996; 243: 9-12.

29 Daras M, Tuchman AJ, Marks S. Central nervous system infarction related to cocaine abuse. Stroke 1991; 22: 1320-13259.

30 Di Lazzaro V, Restuccia D, Oliviero A, Profice P, Nardone R, Valeriani M et al. Ischaemic myelopathy associated with cocaine:clinical, neurophysiological, and neuroradiological features. J Neurol Neurosurg Psychiatry 1997; 63: 531-533.

31 Kalita J, Srivastava AK, Mittal P, Sharma VP, Misra UK. Evoked potential changes in ischaemic myelopathy. Electromyogr Clin Neurophysiol 2003; 43: 211-215.

32 Kawanishi I, Munakata H, Matsumori M, Tanaka H, Yamashita T, Nakagiri K et al. Usefulness of transcranial motor evoked potentials during thoracoabdominal aortic surgery. Ann Thorac Surg 2007; 83: 456-461.

33 Jacobs MJ, Mess W, Mochtar B, Nijenhuis RJ, Statius van Eps RG, Schurink GW. The value of motor evoked potentials in reducing paraplegia during thoracoabdominal aneurysm repair. J Vasc Surg 2006; 43: 239-246.

34 Nogues MA, Pardal AM, Merello M, Miguel MA. SEPs and CNS magnetic stimulation. syringomyelia. Muscle Nerve 1992; 15: 993-1001.

35 Masur H, Oberwittler C, Fahrendorf G, Heyen P, Reuther G, Nedjat S et al. The relation between functional deficits, motor and sensory conduction times and MRI findings in syringomyelia. Electroencephalogr Clin Neurophysiol 1992; 85: 321-330

36 Botzel K, Witt TN. Transcranial cortical stimulation in syringomyelia: correlation with disability? Muscle Nerve 1993; 16: 537-541.

37 Schady W, Dick JPR, Sheard A, Crampton S. Central motor conduction studies in hereditary spastic paraplegia. J Neural Neurosurg Psychiatry 1991; 54: 775-779.

38 Pelosi L, Lanzillo B, Perretti A, Santoro L, Blumhardt L, Caruso G. Motor and somatosensory evoked potentials in hereditary spastic paraplegia. J Neurol Neurosurg Psychiatry 1991; 54: 1099-1102.

39 Manganelli F, Pisciotta C, Dubbioso R, lodice R, Criscuolo C, Ruggiero L et al. Electrophysioligical characterisation in hereditary spastic paraplegia type 5. Clin Neurophysiol 2011; 122: 819-822. 
40 Schüle R, Brandt E, Karle KN, Tsaousidou M, Klebe S, Klimpe S et al. Analysis of CYP7B1 in non-consanguineous cases of hereditary spastic paraplegia. Neurogenetics 2009; 10: 97-104.

41 Sartucci F, Tovani S, Murri L, Sagliocco L. Motor and somatosensory evoked potentials in autosomal dominant hereditary spastic paraparesis (ADHSP) linked to chromosome 2p, SPG4. Brain Res Bull 2007; 74: 243-249.

42 Kalita J, Misra UK. Neurophysiological studies in acute transverse myelitis. J Neurol 2000; 247: 943-948.

43 Leon-Sarmiento FE, Elfakhani M, Boutros NN. The motor evoked potential in AIDS and HAM/TSP: state of the evidence. Arq Neuropsiquiatr 2009; 67: 1157-1163.

44 Di Lazzaro V, Restuccia D, Fogli D, Nardone R, Mazza S, Tonali P. Central sensory and motor conduction in vitamin B12 deficiency. Electroencephalogr Clin Neurophysiol 1992; 84: 433-439.

45 Hemmer B, Glocker FX, Schumacher M, Deuschl G, Lücking CH. Subacute combined degeneration: clinical, electrophysiological, and magnetic resonance imaging findings. J Neurol Neurosurg Psychiatry 1998; 65: 822-827.

46 Utku U, Asil T, Balci K, Uzunca I, Celik Y. Hepatic myelopathy with spastic paraparesis. Clin Neurol Neurosurg 2005; 107: 514-516.

47 Nardone R, Buratti T, Oliviero A, Lochmann A, Tezzon F. Corticospinal involvement in patients with a portosystemic shunt due to liver cirrhosis: a MEP study. J Neuro/ 2006; 253: 81-85.
48 Meyer B, Zentner J. Do motor evoked potentials allow quantitative assessment of motor function in patients with spinal cord lesions? Eur Arch Psychiatry Clin Neurosci 1992; 241: 201-204.

49 Zentner J, Rieder G. Diagnostic significance of motor evoked potentials in spaceoccupying lesions of the brain stem and spinal cord. Eur Arch Psychiatry Neurol Sci 1990; 239: 285-289.

50 Lissens MA, Vanderstraeten GG. Motor evoked potentials of the respiratory muscles in tetraplegic patients. Spinal Cord 1996; 34: 673-678.

51 Zifko U, Remtulla H, Power K, Harker L, Bolton CF. Transcortical and cervical magnetic stimulation with recording of the diaphragm. Muscle Nerve 1996; 19: 614-620.

52 Curt A, Dietz V. Electrophysiological recordings in patients with spinal cord injury:significance for predicting outcome. Spinal Cord 1999; 37: 157-165.

53 Curt A, Keck ME, Dietz V. Functional outcome following spinal cord injury: significance of motor-evoked potentials and ASIA scores. Arch Phys Med Rehabil 1998; 79. 81-86.

54 Petersen JA, Spiess M, Curt A, Dietz V, Schubert MEM-SCI Study Group. Spinal cord injury: one-year evolution of motor-evoked potentials and recovery of leg motor function in 255 patients. Neurorehabil Neural Repair 2012; 26: 939-948.

55 van Hedel HJ, Murer C, Dietz V, Curt A. The amplitude of lower leg motor evoked potentials is a reliable measure when controlled for torque and motor task. J Neurol 2007; 254: 1089-1098. 